Dear Author,

Please, note that changes made to the HTML content will be added to the article before publication, but are not reflected in this PDF.

Note also that this file should not be used for submitting corrections. 


\section{AUTHOR QUERY FORM}

\begin{tabular}{|c|c|c|}
\hline 1.t. & Journal: SNA & $\begin{array}{l}\text { Please e-mail or fax your responses and any corrections to: } \\
\text { E-mail: corrections.esch@elsevier.thomsondigital.com }\end{array}$ \\
\hline ELSEVIER & Article Number: 8966 & Fax: +35361709272 \\
\hline
\end{tabular}

Dear Author,

Please check your proof carefully and mark all corrections at the appropriate place in the proof (e.g., by using on-screen annotation in the PDF file) or compile them in a separate list. Note: if you opt to annotate the file with software other than Adobe Reader then please also highlight the appropriate place in the PDF file. To ensure fast publication of your paper please return your corrections within 48 hours.

For correction or revision of any artwork, please consult http://www.elsevier.com/artworkinstructions.

Any queries or remarks that have arisen during the processing of your manuscript are listed below and highlighted by flags in the proof. Click on the 'Q' link to go to the location in the proof.

\begin{tabular}{|l|l|}
\hline $\begin{array}{c}\text { Location in } \\
\text { article }\end{array}$ & \multicolumn{1}{c}{$\begin{array}{c}\text { Query / Remark: click on the Q link to go } \\
\text { Please insert your reply or correction at the corresponding line in the proof }\end{array}$} \\
\hline Q1 & $\begin{array}{l}\text { Please confirm that given names and surnames have been identified correctly. } \\
\text { Q3 }\end{array}$ \\
Q4 & $\begin{array}{l}\text { As per style, author photograph is mandatory. Please provide the same for both the authors. } \\
\text { Fig. 11 will appear in black and white in print and in color on the web. Based on this, the respective } \\
\text { figure caption has been updated. Please check, and correct if necessary. }\end{array}$ \\
& $\begin{array}{l}\text { Please check this box or indicate your approval if } \\
\text { you have no corrections to make to the PDF file }\end{array}$ \\
\hline
\end{tabular}

Thank you for your assistance. 


\section{A MEMS linear accelerator for levitated micro-objects}

Ibrahim Sari*, Michael Kraft

- We present a contactless linear accelerator.

- Electromagnetic levitation and electrostatic propulsion techniques are used and this is the first time in literature that such a hybrid design is used.

- It is capable of levitating and accelerating objects on a defined path.

- A proof of concept design is fabricated and tested for performance.

- Tests showed a maximum levitation height of $75 \mu \mathrm{m}$ and a maximum velocity of $3.6 \mathrm{~mm} / \mathrm{s}$ can be achieved. 


\title{
A MEMS linear accelerator for levitated micro-objects
}

\author{
Q1 , Jbrahim Sari*, Michael Kraft \\ University of Southampton, School of Electronics and Computer Science, Nano Group, Southampton SO17 1BJ, United Kingdom
}

\section{A R T I C L E I N F O}

\section{Article history:}

Received 22 April 2013

Received in revised form 29 October 2014

Accepted 15 November 2014

Available online $\mathrm{xxx}$

\section{Keywords:}

Microactuators

Linear accelerator

Micro-injector

Electromagnetic levitation

Electrostatic propulsion

\begin{abstract}
A B S T R A C T
In this work the design, micro-fabrication, and characterization steps of a contactless linear accelerator is presented. The proposed design in principle can levitate conductive micro-objects and accelerate or move them over a predefined trajectory. The levitation is realized using electromagnetic induction generated by a changing AC field whereas the propulsion is achieved through electrostatic forces from a controlled DC source. This is the first time in literature that such a hybrid design is used to accomplish this idea. It has been experimentally shown that the proposed design can levitate $1 \mathrm{~mm} \times 1 \mathrm{~mm}$ sized and $7 \mu \mathrm{m}$ thick micro-objects to a maximum height of $75 \mu \mathrm{m}$ and propel them forward continuously at a maximum average forward velocity of $3.6 \mathrm{~mm} / \mathrm{s}$.
\end{abstract}

(C) 2014 Published by Elsevier B.V.

\section{Introduction}

Following the recent advances in the microsystems technology, high-performance micro-inertial actuators and sensors can now be fabricated using standard fabrication methods and thus are widely available in a range of specifications. Although being easily accessible, the performance of such devices is still limited by a number of constraining factors such as proof mass weight, spring constant, and feature size. Among these factors, one important physical limitation is that the sensing or actuating element that is the proof mass, is constrained by a suspension structure acting as a mechanical spring. This limitation can degrade the overall performance of these devices such as sensitivity or output force, or restrict the motion range of an actuator. One way to overcome such limitation is to use other means to support the proof mass rather than using physical connections. For this purpose, in various studies so far, the sensing or the actuation element was supported through levitation using electromagnetic and electrostatic levitation techniques. Some applications examples used for this purpose were contactless micro-motors, gyroscopes, and micro-bearings.

This study presents a novel micro-linear manipulator, which can levitate and accelerate conductive micro-objects and extends the preliminary results outlined in [1]. Previously, Shearwood et al. [2] demonstrated a levitated and rotating gyroscope. In their work, the design featured a circular aluminum track over which an aluminum

\footnotetext{
* Corresponding author. Tel.: +44 2380593127

E-mail address: is2@ecs.soton.ac.uk (I. Sari).
}

rotor was levitated and rotated by electromagnetic (EM) induction and a rotating EM field, respectively. The levitated rotor was constraint by stabilizing EM forces that were generated by a planar metal loop acting as stability coil. In a related later study by $\mathrm{Wu}$ et al. [3], similar techniques were used to levitate, rotate, and stabilize a rotor for the application of a gyroscope. In another study by Murakoshi et al. [4], a levitated, ring shaped rotational gyroscope and accelerometer is presented. Electrostatic (ES) forces were used to levitate, stabilize, and rotate the rotor. Different from the previous two studies, a closed-loop force feedback technique is required for the position control of the rotor. In addition to the work discussed so far, similar EM and ES levitation and control techniques were presented for other applications as well such as micro-motors and micro-bearings [5-9]. Besides these, Kumar et al. demonstrated a levitated linear actuator where a $24 \mathrm{~mm} \times 24 \mathrm{~mm} \times 180 \mu \mathrm{m}$ sized object was first levitated by ES forces. Then, the levitated object was moved forward and backwards by a stroke of $200 \mu \mathrm{m}$ by switching the drive electrodes on and off, respectively [10]. Another type of levitated linear actuator was presented by Jin et al. [11]. In this work, an 8-in Si wafer was levitated by electrostatic forces and propelled along a linear path by sequentially energizing pairs of electrodes. This was a macro scale application, which was mainly developed for contactless transfer of silicon wafers. Different from active levitation strategies, Liu et al. [12] presented a levitated diamagnetic rotor that was levitated by NdFeB magnets. The levitated rotor was then actuated by a rotating ES field similar to an axial variable-capacitance motor.

A number of techniques have been demonstrated to levitate and manipulate micro-objects in various applications. However, 


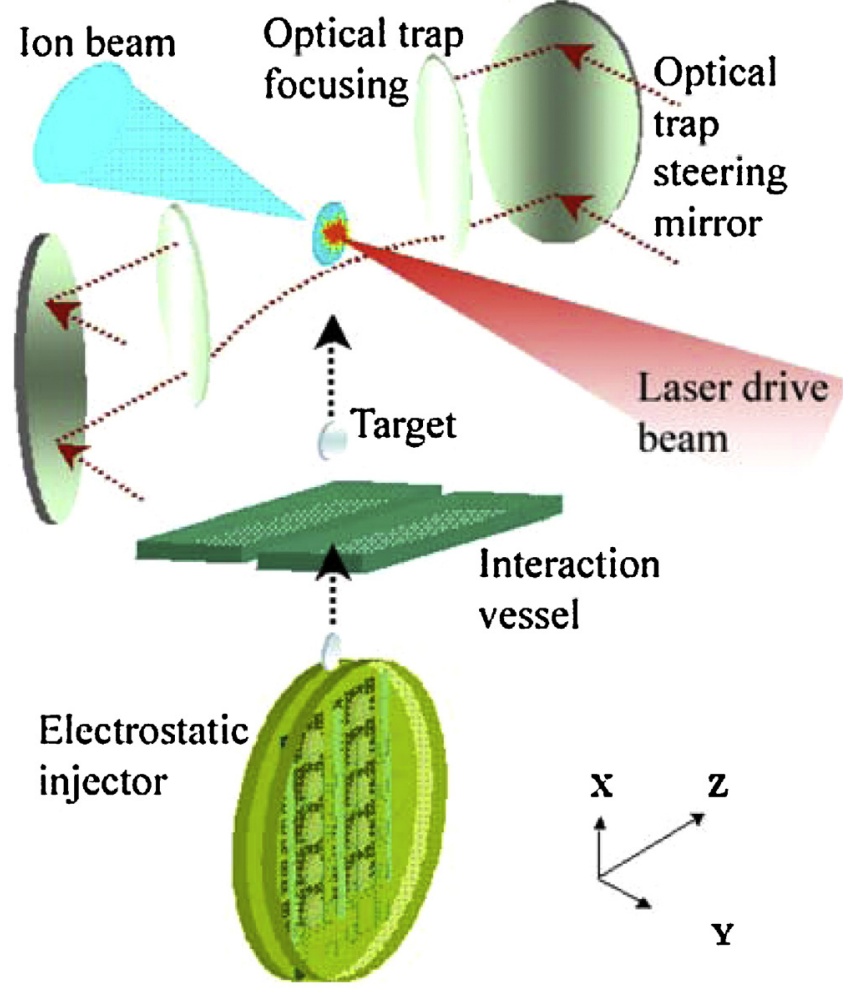

Fig. 1. Schematic representation of the application where the proposed manipulator is intended to be used.

in most of these applications, circular micro-objects were manipulated and most importantly they did not have any continuous propulsion capability along a linear trajectory. In this work, the proposed design is capable of both levitating and accelerating micro-objects along a predefined path. It combines two different techniques to realize this; electromagnetic induction for levitation and electrostatic forces for propulsion. It can be used in a number of applications like contactless acceleration and positioning of micro-objects, injection systems, and contactless inertial sensors. The design presented here was specifically developed for a target injection and trapping application. Fig 1 is a schematic representation of the final application where the proposed device is aimed to be used. It is composed of a vacuum interaction vessel and an electrostatic injector system (note that the actual levitation and injection system design proposed in this work is schematically different than the "electrostatic injector" shown in this figure). There is an optical trapping system [13], a laser drive beam, and measurement instruments inside the interaction vessel. The micro-object of interest is levitated and accelerated inside the vacuum interaction vessel using the design presented in this work after which it is trapped by the optical trapping system. The levitated object is then moved to an optimal position where it is shot by a laser beam at different power levels and intensities and the resulting ionbeam radiation is investigated. For this application, it is crucial that the micro-objects are suspended without any attachments as such connections would perturb the ion-beam production mechanism. The investigation of resulting ion-beam production from different materials has importance in many fields such as:

- medicine (cancer treatment through laser energized protons and ions at a much lower cost),

- industry (in situ radiography, diagnostics, semiconductor fabrication),

- science (research in synchronized beam production),

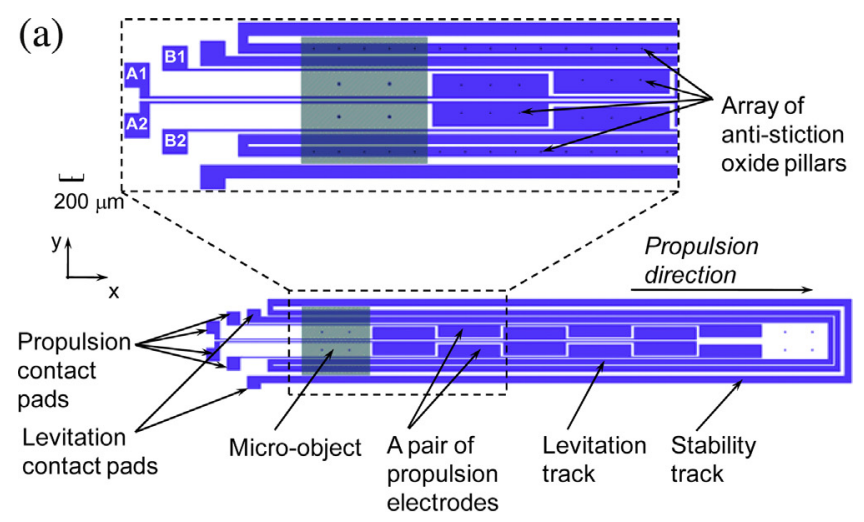

(b)

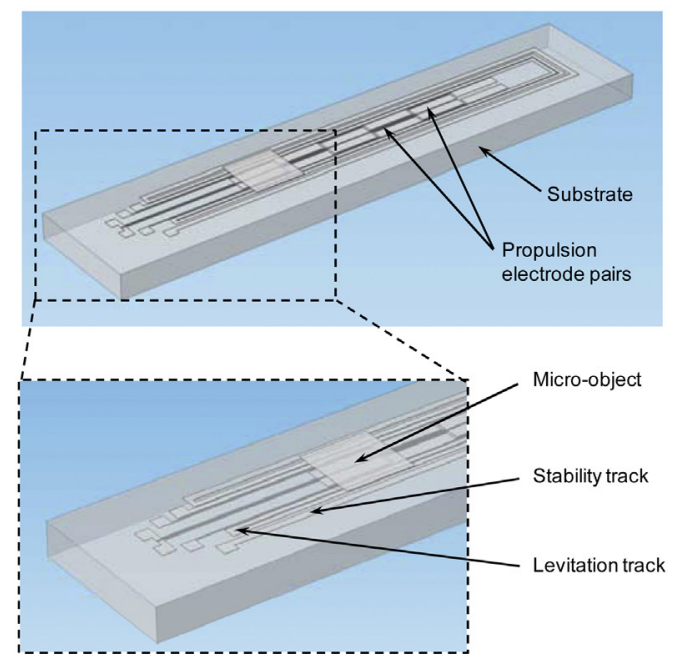

Fig. 2. (a) Schematic of the proposed micro-linear manipulator that is composed of levitation and stability tracks and forward propulsion electrodes. (b) Isometric view of the design.

- security (image detection of hidden materials and objects using tomography).

To the best of authors' knowledge, this is the first time in literature that a combined levitation and acceleration design concept is proposed and demonstrated to work. The paper is organized as follows: Section 2 describes the modeling, and simulation, Section 3 discusses the fabrication steps of the design, and finally Section 4 presents the experimental results of the work together with actual measurement data for levitation and propulsion.

\section{Design}

Fig. 2a shows a schematic top view of the device prototype comprising an outer rectangular, conductive aluminum loop and a series of paired rectangular aluminum electrodes inside this loop. Fig. $2 \mathrm{~b}$ is an isometric view of the design. The micro-object is levitated by electromagnetic induction by applying an AC signal $(10-55 \mathrm{MHz})$ to the electrical pads of the rectangular loop. The loop consists of an inner (levitation) and an outer (stability) track. The inner track induces a levitation force on the micro-object that is defined by the Lorentz force:

$\vec{F}=\vec{J} \times \vec{B}$

where $F$ is the levitation force exerted on the object, $B$ is the magnetic field density induced by the alternative current in the inner track and $J$ is the induced eddy current density in the object by the magnetic field. It would ideally be beneficial to derive a detailed 


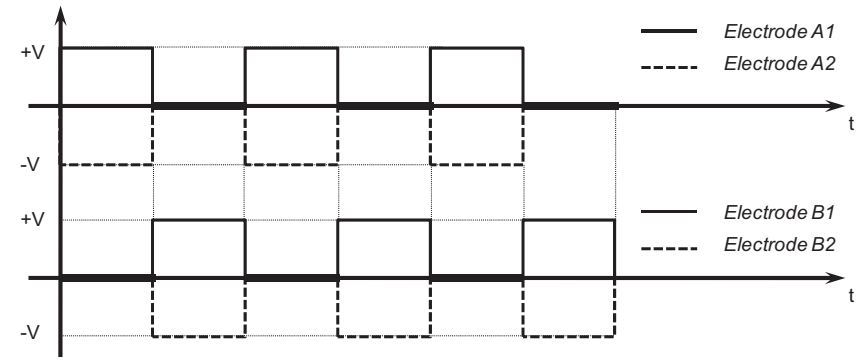

Fig 3. Relative drive voltage sequence and polarity of propulsion electrode sets energized through pads A1-2 and B1-2.

expression in order to represent the dynamics and forces on the micro-object. However, it is not possible to find such a closed form expression due the three dimensional complex geometry of the design. For this purpose a detailed Comsol FEM model is constructed to fully represent the dynamics of the proposed design instead of using an over simplified mathematical model of the design.

The outer track provides lateral stability and generates an inward electromagnetic force keeping the levitated object in the mid-position along the $x$-axis. This force is due to the interaction between the induced currents in the object and the electromagnetic field due to the current in the loop. If an external force moves the object away from the y-origin then the object always experiences a counteracting force, which tries to bring it back to the original equilibrium position. A similar stability feature was previously reported for the applications of levitated gyroscopes and micro-motors $[1,3]$. In this work this feature is successfully extended to a linear track.

In order to reduce the overall contact area between the object at rest and the electrodes, and decrease the initially required levitation force, oxide pillars are fabricated on top of the electrodes. They are also used as an electrically insulating medium.

After levitation, the object is propelled forward by applying a square wave voltage pattern to the propulsion electrodes. These electrodes act as a parallel plate capacitor and cause two electrostatic force components on the micro-object; a forward driving force and a normal attraction force. The forward driving force moves the levitated object forward accelerating it whereas the normal attraction force pulls the object vertically downwards reducing the levitation height. The latter is an unwanted effect and has been considered during Comsol simulations.

In order to maintain a continuous forward propulsion force, each pair of adjacent electrodes is energized sequentially with a phase difference of $180^{\circ}$. with respect to the neighboring electrodes pair. The phase difference of $180^{\circ}$ between the neighboring electrodes is to create a net forward propulsion force. When one set of electrodes, for example A1 and A2 is activated, the neighboring electrodes B1 and B2 are deactivated. This creates a net forward force on the micro-object that causes it to move along the track. If both sets of electrodes were activated at the same time i.e. if there were no phase difference, then the object would be attracted equally in $+x$ and $-x$ directions (see Fig. $2 a$ for sign conventions) such that it would stand still due to equal and opposite forces applied at the same time. This is illustrated in Fig 3 , which shows the relative voltage sequence and polarity of each electrode that are energized through the pads A1, A2, B1, and B2 (see Fig. 2a). In this particular design six electrode pairs are fabricated, but depending on the application this could be arbitrarily adjusted. The electrode sets A and $B$ are symmetric in forward and backward directions and hence without knowing the initial conditions of the object i.e. the initial position and velocity, it would be uncertain to know which direction the object would move. For this reason, in order to assure that

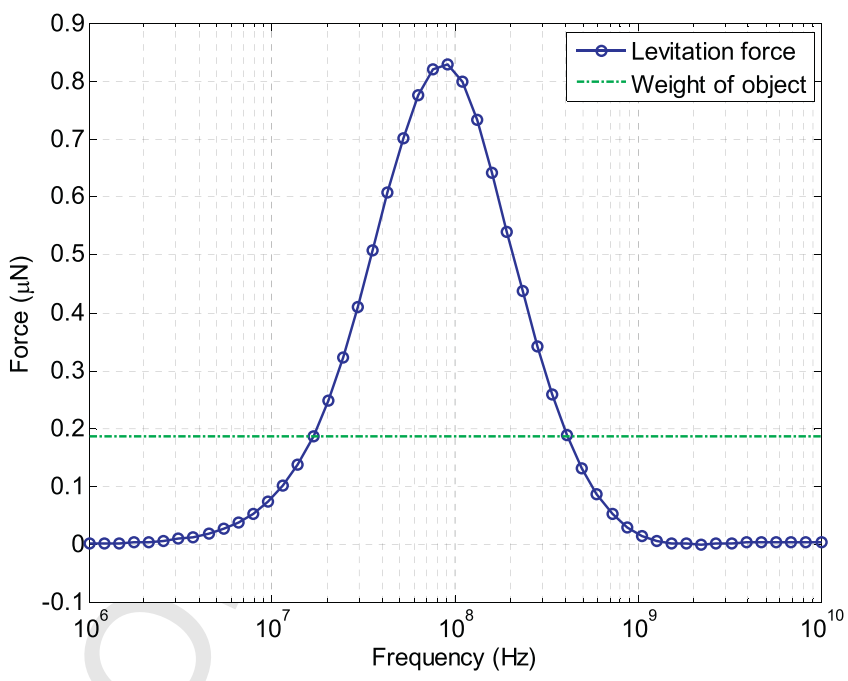

Fig. 4. COMSOL finite element (FEM) simulation of the electromagnetic levitation force with respect to excitation frequency.

the object always moves forward, it is always started to be levitated and propelled from the first pads as illustrated Fig ${ }_{\wedge} 2 \mathrm{a}$.

The design was simulated in Comsol using the design parameters tabulated in Table 1 . The electrodes are fabricated from aluminum due to its relatively high conductivity, which becomes important at high frequencies due to the skin effect.

Fig. 4 shows the resulting lift force on the object with respect to frequency for a peak current amplitude of $0.6 \mathrm{~A}$ and a levitation height of $30 \mu \mathrm{m}$. The weight of the object is also shown for comparison purposes.

From Fig 4 4, the theoretically predicted feasible frequency range to achieve levitation is $15-400 \mathrm{MHz}$. The levitation force has a maximum at about $90 \mathrm{MHz}$, afterwards it decreases due to the skin effect. This is an important parameter to be considered when working at relatively high frequency ranges. It is mainly an indication of high frequency resistance of a conductor and can be expressed in terms of the skin depth of a conductor at a specific frequency. The current density in the conductor decreases exponentially with the depth $\mathrm{d}$ from the surface for a semi-infinite conductor:

$J=J_{S} e^{-d / \delta}$

where $J$ is the current density at a depth d from the surface, $J_{S}$ is the current density at the surface i.e. $J_{S}=J(0)$, and $\delta$ is skin depth of the conductor at the frequency of interest and defined as:

$\delta=\sqrt{\frac{2 \rho}{\omega \mu}}$

where $\rho$ is the resistivity of the conductor, $\omega$ is the AC current frequency, and $\mu$ is the absolute magnetic permeability of the

Table 1

Important properties of the design.

$\begin{array}{ll}\text { Material (electrodes and micro-object) } & \text { Aluminum } \\ \text { Overall device dimensions } & 9.5 \mathrm{~mm} \times 1.2 \mathrm{~mm} \times 0.5 \mathrm{~mm} \\ \text { Stability and levitation track width } & 80 \mu \mathrm{m} \\ \text { Propulsion electrode dimensions } & 900 \mu \mathrm{m} \times 200 \mu \mathrm{m} \times 7 \mu \mathrm{m} \\ \text { Dimensions of oxide pillars (diameter } \times \text { thickness) } & 8 \mu \mathrm{m} \times 1 \mu \mathrm{m} \\ \text { Size of the micro-object } & 1 \mathrm{~mm} \times 1 \mathrm{~mm} \\ \text { Thickness of micro-object } & 7 \mu \mathrm{m} \\ \text { Impedance of levitation track } & 4 \Omega \\ \text { Total power consumption } & 80 \mathrm{~mW} \\ \text { Levitation signal frequency } & 10-50 \mathrm{MHz} \\ \text { Levitation peak current amplitude } & 0.2-0.7 \mathrm{~A} \\ \text { Propulsion voltage frequency } & 0.1-4 \mathrm{~Hz} \\ \text { Propulsion voltage amplitude } & 4-9 \mathrm{~V}(\text { square wave) }\end{array}$




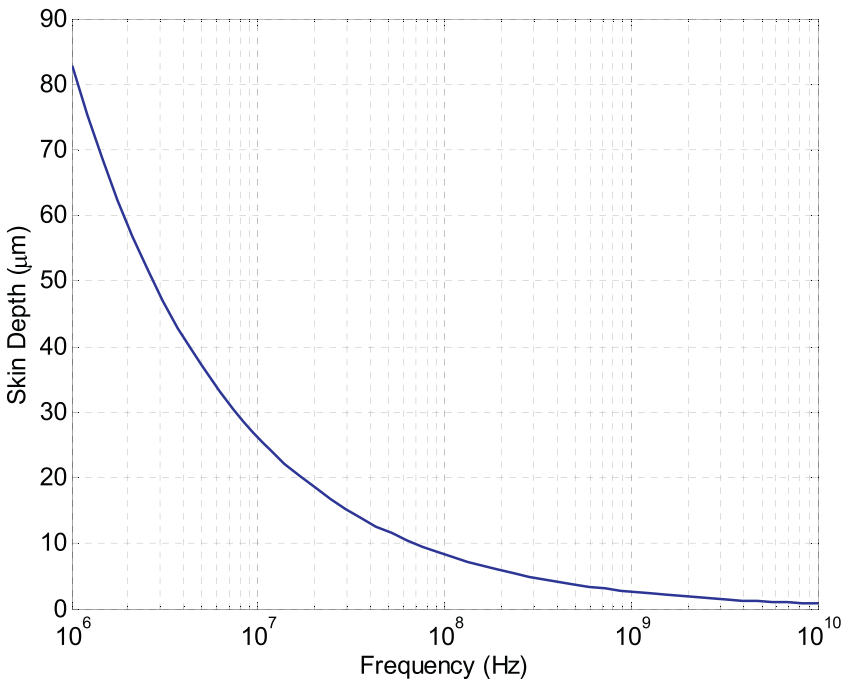

Fig 5. Plot showing the variation of skin depth with frequency for aluminum. For a $7 \mu \mathrm{m}$ thick conductor, the current density would start decreasing at a frequency smaller than $140 \mathrm{MHz}$. This is the consistent with the levitation force simulation where the levitation force is making a maximum at about $90 \mathrm{MHz}$.

conductor. Fig. 5 shows the variation of skin depth with frequency for aluminum that has a resistivity of $\rho=2.7 \times 10^{-8} \Omega \mathrm{m}$ and absolute magnetic permeability of $\mu=4 \pi \times 10^{-7} \mathrm{H} / \mathrm{m}$.

It follows from Eq. (2) that the current density drops to $1 / e$ (about 37\%) of its initial value at a depth from the surface of the conductor that is equal to the skin depth. So from the skin depth plot, for a $7 \mu \mathrm{m}$ thick conductor, which is the same thickness of the designed value, the current density would be $1 / e$ of its initial value at a frequency of $140 \mathrm{MHz}$. This means that the levitation force, which is proportional to the current density, would normally start decreasing i.e. make a maximum at a frequency much smaller than $140 \mathrm{MHz}$. This is consistent with the levitation force simulation where the force is having a maximum at around $90 \mathrm{MHz}$. In order to further support this argument, Fig, 6 shows the variation of the non-dimensional current density with respect to non-dimensional depth. The non-dimensional current density is the percentage current density from the surface of the conductor to its nominal value

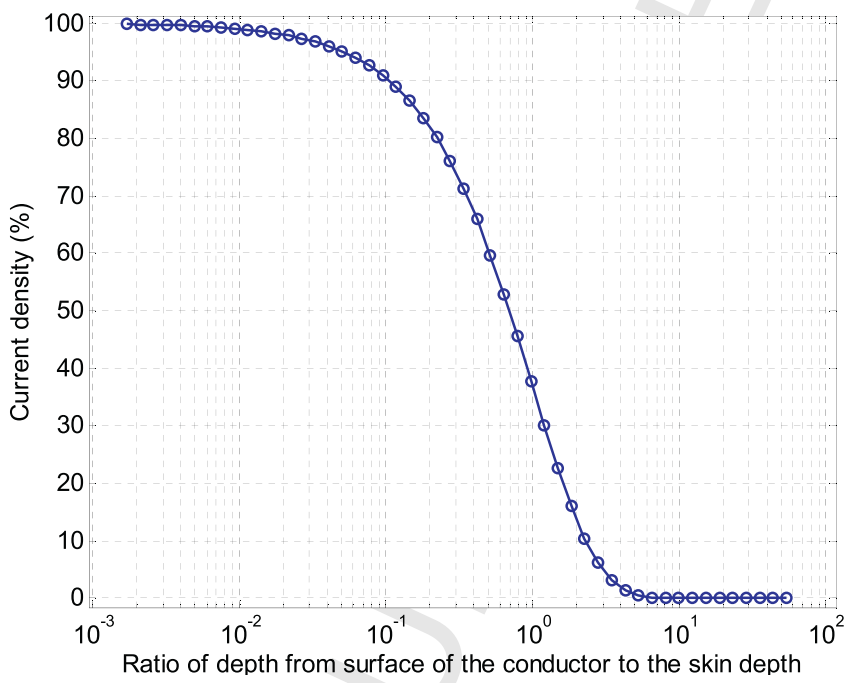

Fig 6. Variation of non-dimensional current density with respect to nondimensional depth. The non-dimensional current density is the percentage current density from the surface of the conductor to its nominal value at the surface, and the non-dimensional depth is the ratio of the depth where the current density is calculated to the skin depth.
Fabrication flow of the levitation design
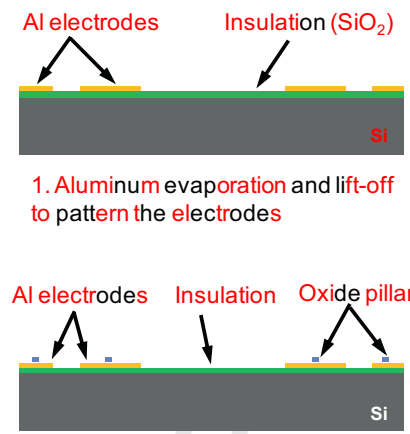

2. Oxide evaporation and lift-off to form the pillars on top of Al electrodes.

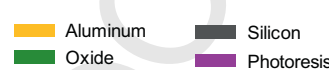

Fig. 7. Fabrication flow of the levitation system (left) and the micro-object (right).

at the surface, and the non-dimensional depth is the ratio of the depth where the current density is calculated to the skin depth. It follows from $\mathrm{Eq}_{\lambda}(2)$ that this can be interpreted as $J / J_{S}$ vs $d / \delta$. From the figure, it can be concluded that at only a depth of 0.7 times the skin depth, the current density drops down to $50 \%$ of its value at the surface.

The main design parameters other than the thickness of the micro-object are width and height of the micro-object, thickness of the tracks, and dimension of the oxide pillars. The lateral dimensions of the micro-object are selected to be $1 \mathrm{~mm} \times 1 \mathrm{~mm}$ considering easiness of handling and levitation. Smaller $(0.5 \mathrm{~mm} \times 0.5 \mathrm{~mm})$ and larger $(1.5 \mathrm{~mm} \times 1.5 \mathrm{~mm})$ sized objects has also been fabricated and tested with corresponding device designs. Smaller ones were difficult to handle manually as they were too small for the vacuum tweezer to pick up. Larger ones were much harder to levitate and manipulate and propel forward due to their larger size and weight. These could only be verified after fabrication and during the tests as it is not possible to simulate such effects beforehand. The preliminary size selection was mainly based on the previous work carried out in the literature.

The thickness of the tracks is chosen to be $7 \mu \mathrm{m}$ to have a high current carrying capability and reduce the track resistance. It could be made thicker, but this would make the fabrication much harder using the standard fabrication methods.

The oxide pillars are designed to be $1 \mathrm{um}$ high to have enough clearance between the object and the aluminum surface so that the risk of stiction would be reduced.

\section{Fabrication}

The devices and the micro-objects are fabricated using a two-mask fabrication process. Fig. 7 shows the corresponding fabrication flow where the fabrication of the devices and the microobject are shown to the left and right of the figure, respectively. The devices are fabricated over a silicon wafer that is coated by $200 \mathrm{~nm}$ of silicon dioxide to electrically insulate the electrical tracks from the substrate. The silicon wafer is first patterned by $14 \mu \mathrm{m}$ of AZnLOF 2070 type negative tone photoresist from Microchemicals $\mathrm{GmbH}$ [14]. This resist is specially designed for lift-off since the sidewall profile can be easily controlled by the exposure dose and the development rates. Having a negative sidewall profile provides the thick metal layers to be easily lifted off. After resist patterning, $7 \mu \mathrm{m}$ thick aluminum is deposited on the substrate by e-beam 
evaporation to form the electrodes, contact pads, and levitation tracks. After metal deposition, the unnecessary metal areas are lifted off in NMP (1-methyl-2-pyrrolidone) solvent. This is a powerful lift-off medium and avoids the lifted metal layers sticking to each other or back on the substrate due to its oily character [15]. It is then followed by the fabrication of $8 \mu \mathrm{m}$ diameter and $1 \mu \mathrm{m}$ thick anti-stiction oxide pillars on top of the electrodes by a similar procedure, but this time by silicon dioxide e-beam evaporation and lift-off. Afterwards, the fabricated devices are rinsed and coated by a protective photoresist layer and diced into chips. Finally, the protective resist layer is removed in an oxygen plasma asher and wirebonded to a PCB for testing.

The $7 \mu \mathrm{m}$ thick and $1 \mathrm{~mm} \times 1 \mathrm{~mm}$ sized micro-objects are in house fabricated as well using a very similar procedure to device fabrication that is by aluminum evaporation and lift-off. The main difference is that, the substrate is blanket coated by a $2 \mu \mathrm{m}$ thick AZ520D type protective coating as a sacrificial layer before the negative photoresist patterning step. This coating is not photosensitive and does not create nitrogen to be formed during exposure of the negative photoresist and it can also be dissolved using the NMP solvent [16]. Hence, using this sacrificial layer, micro-objects and the rest of the dummy metal lines are floating in the lift-off NMP medium at the end of the lift-process. The micro-objects are then picked up from the solution using a syringe with a tip opening of $0.5 \mathrm{~mm}$, rinsed by isopropyl alcohol, and finally dried by nitrogen.

Fig, 8 (left) shows an image of a fabricated device; the lighter colored areas represent the device features and the darker base is the Silicon substrate deposited with a thin insulation layer. To the top right of the figure, the SEM image of a part of the $80 \mu \mathrm{m}$ wide electrical tracks and a group of $30 \mu \mathrm{m}$ diameter and $7 \mu \mathrm{m}$ thick aluminum posts are shown. A detail SEM image of one of these posts is shown on the bottom right hand side of the figure; they are used to support the $10 \mu \mathrm{m}$ diameter and $1 \mu \mathrm{m}$ thick silicon dioxide pillars fabricated on the top. It is crucial to use these pillars (i) to insulate the micro-object from the electrical tracks and more importantly (ii) to reduce the initial levitation force by reducing the contact area between the micro-object and the surface where it is initially resting on.

Fig 9 shows a photograph of a fabricated and diced chip that is wire bonded to a PCB and ready for testing. In this figure, there are four devices with a micro-object placed on the leftmost device using vacuum tweezers.

\section{Characterization}

Fig 10 shows the block diagram representation of the test setup that was used to characterize the fabricated devices. An RF signal generator and amplifier were used to induce a lift force and hence levitate the micro-objects. Once the object was levitated a square wave generator and a custom circuitry were used to propel the object forward. The levitation and propulsion characteristics of the design were measured using a white light interferometer and a camera, respectively.

Fig $11 \mathrm{a}$ and $\mathrm{b}$ shows microscope images of a part of the device with the micro-object levitated under a white light interferometer; in Fig, 11a, the levitation tracks are in focus and the object is blurred whereas in Fig 11b, the object is in focus and the levitation tracks are blurred. These two images were taken with the same focal setting. The focal difference of the Al plate proves that levitation is successfully achieved. Fig $11 \mathrm{c}$ shows the levitation height measurement that is taken using a white light interferometer (only approximately a third of the width of the Al plate could be shown as this was the maximum field of view of the microscope). The figure depicts a color-coded height plot of the device features and the levitated object for the same configuration as of Fig $11 \mathrm{a}$ and

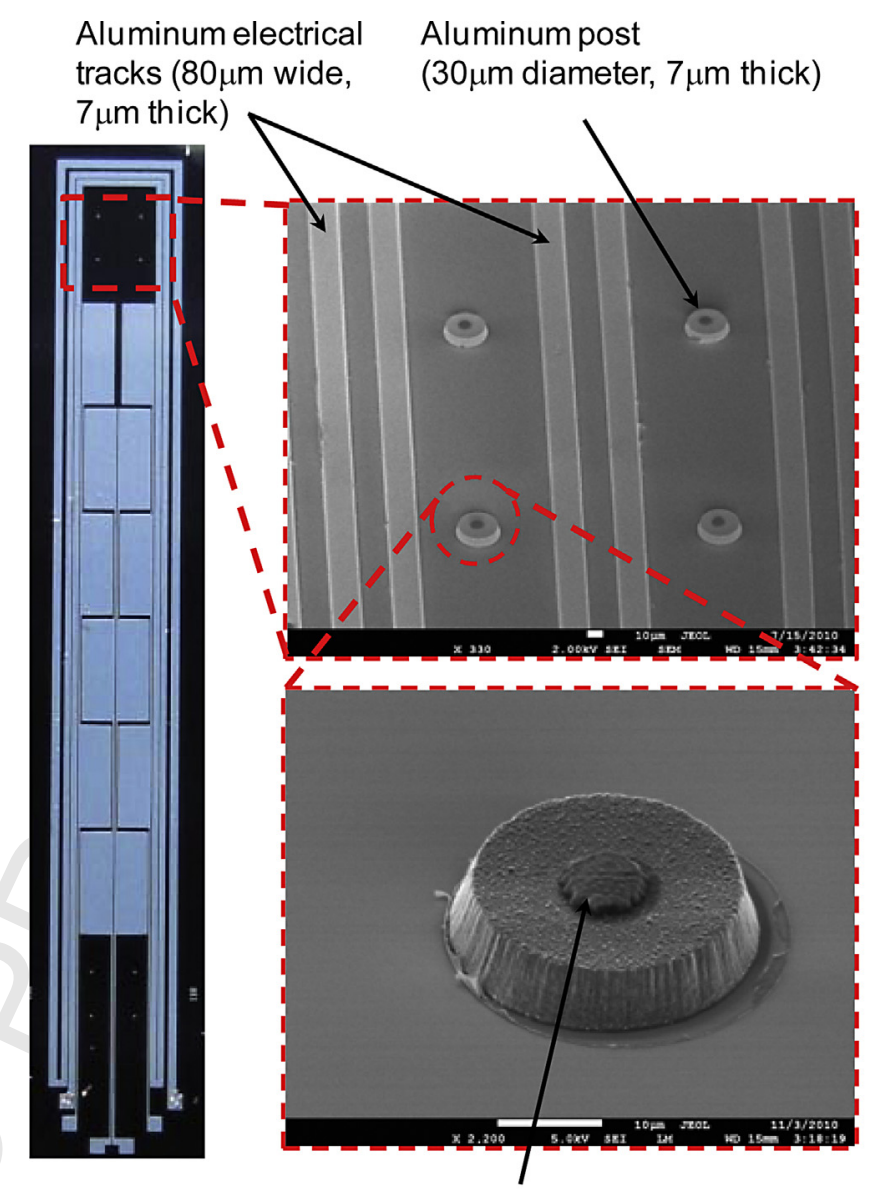

Device (light) over Silicon dioxide pillar $(10 \mu \mathrm{m}$ diameter, a substrate (dark) $1 \mu \mathrm{m}$ thick) over an aluminum post

Fig. 8. Image of an individual device fabricated on an insulated silicon substrate (left). A close-up SEM image of the electrical tracks and aluminum posts are shown on the upper right. They are used to support the silicon dioxide pillars that are used to insulate the micro-object from the electrical tracks and reduce the contact area between the micro-object and the surface where it is initially resting on (detail SEM view shown on the bottom right).

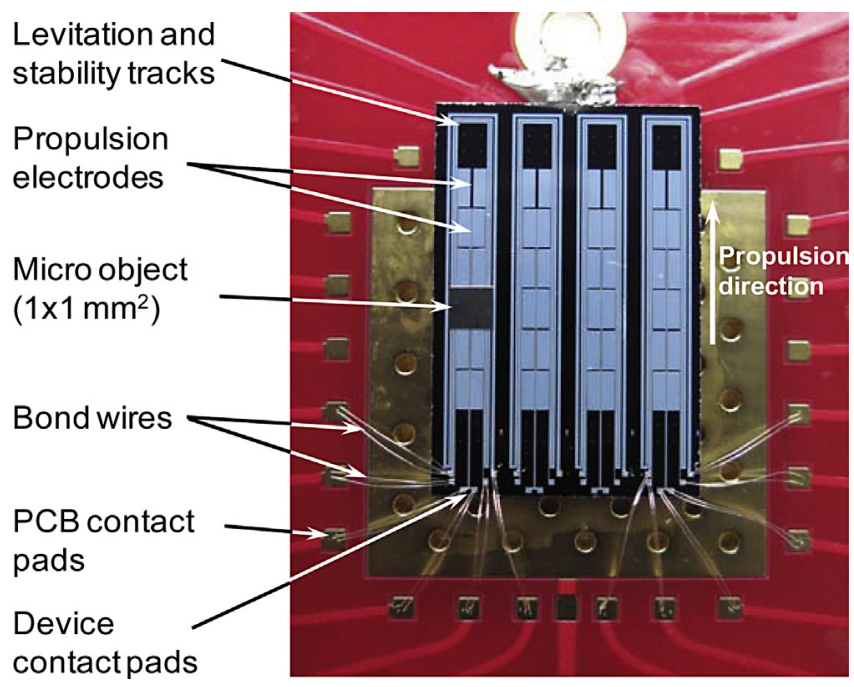

Fig. 9. Photograph of a fabricated chip wire bonded to a PCB and ready for testing. 


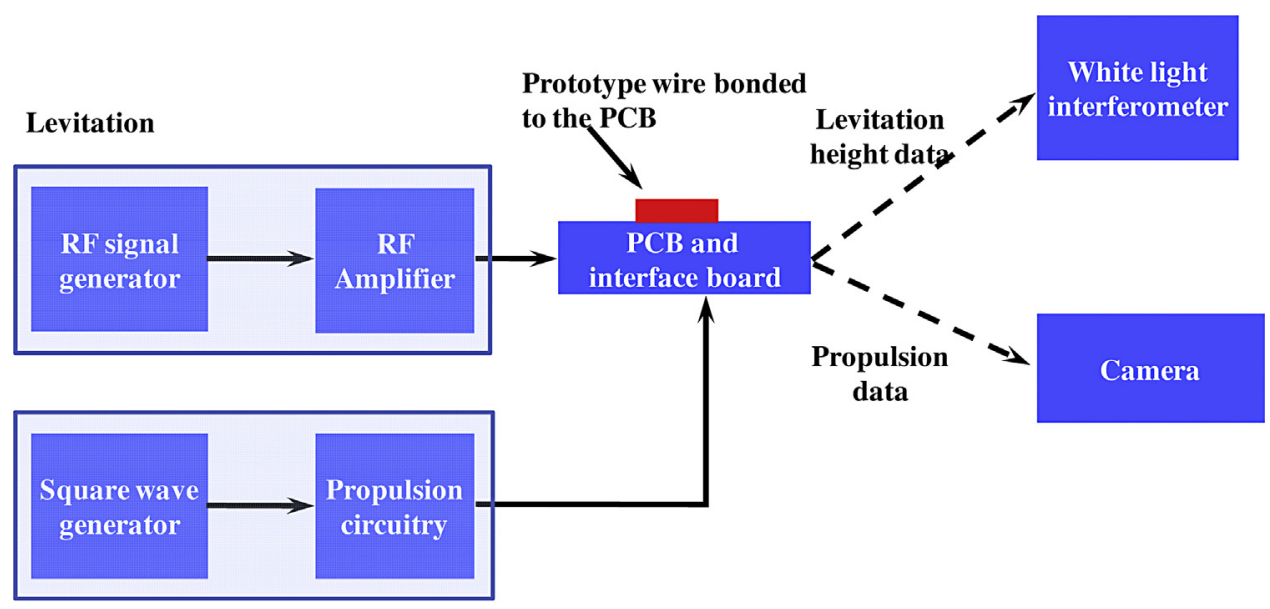

\section{Propulsion}

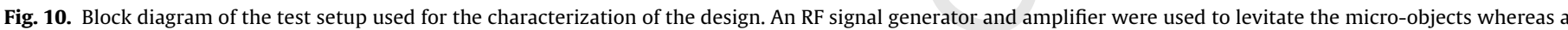
square wave generator together with a custom made circuitry were used to drive the levitated objects forward.

b. Despite the limited field of view it, the uniformity of the color of the levitated object (in blue) is a clear indication for the flatness of the levitated object and that the plate is levitated parallel to the substrate.
Fig 12 shows the levitation height with respect to frequency of the input AC signal for a peak current amplitude of $0.6 \mathrm{~A}$ through the levitation tracks. The levitation height is measured for each frequency value using a white light interferometer. The levitation

\section{(a)}

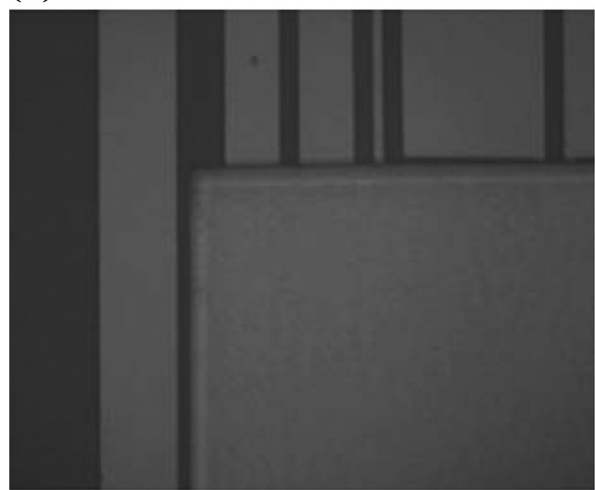

(b)

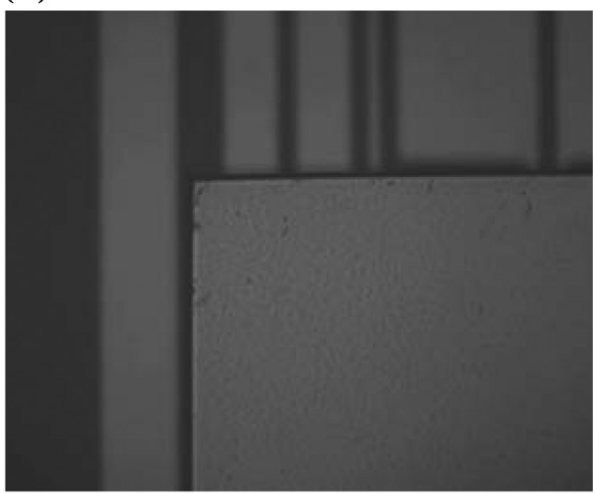

(c)

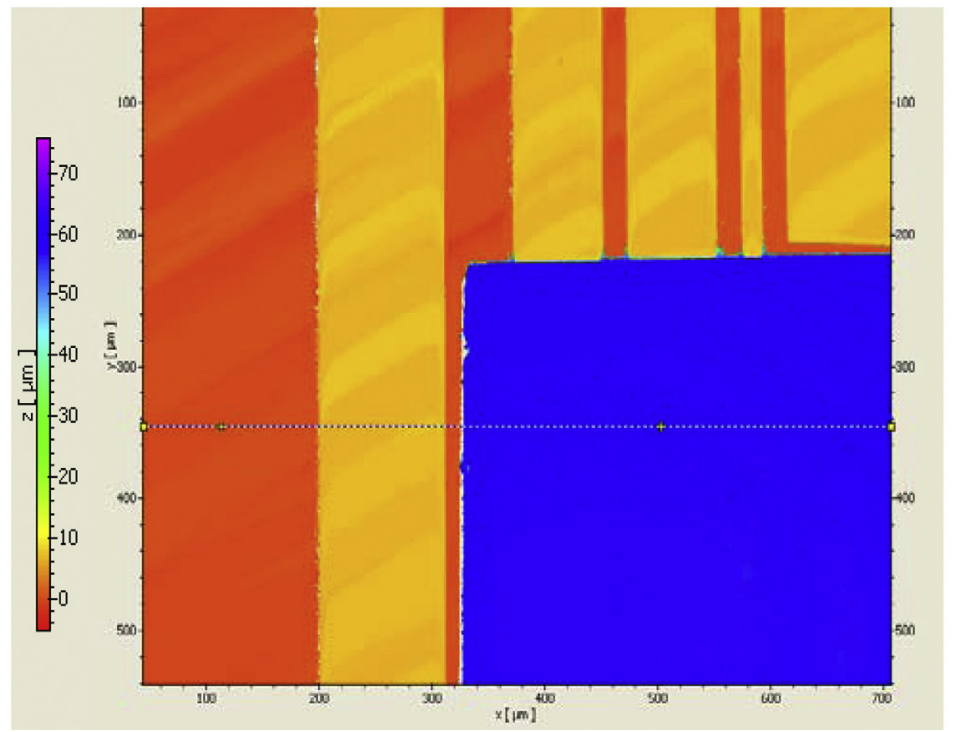

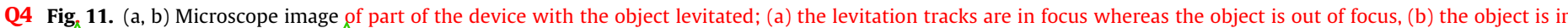

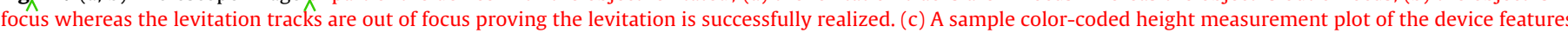

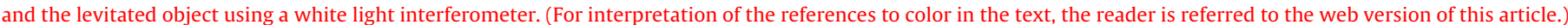




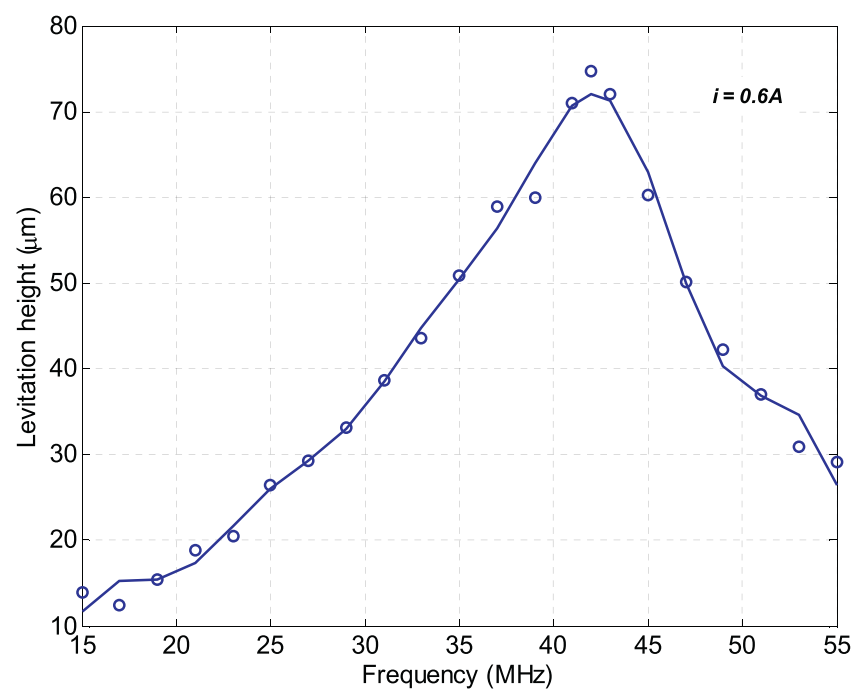

Fig. 12. Measured variation of levitation height with respect to the frequency of the input signal for a peak input current amplitude of $0.6 \mathrm{~A}$.

height is increasing with frequency until $42 \mathrm{MHz}$ where it reaches a peak value of $75 \mu \mathrm{m}$ and decreases afterwards. This is due to the skin effect, which is also observed in the FEM simulations of levitation force.

Fig 13 shows the measured (circle marked line) and simulated (square marked line) levitation height with respect to the supply current for an excitation frequency of $35 \mathrm{MHz}$. The levitation height increases with the supply current, but with a decreasing slope. This is also observed in previous studies [1] and is due to the inverse relation of the levitation force with the levitation height. There is a maximum deviation of $5 \%$ between the measured and simulation values. This is mainly considered to be caused by the contact and line resistances on the PCB board on which the fabricated chip is mounted.

The propulsion feature of the design was tested by using a custom made analog circuit that can be represented by the block diagram shown in Fig 14 . The circuit sequentially energizes each pair of adjacent propulsion electrodes such that they

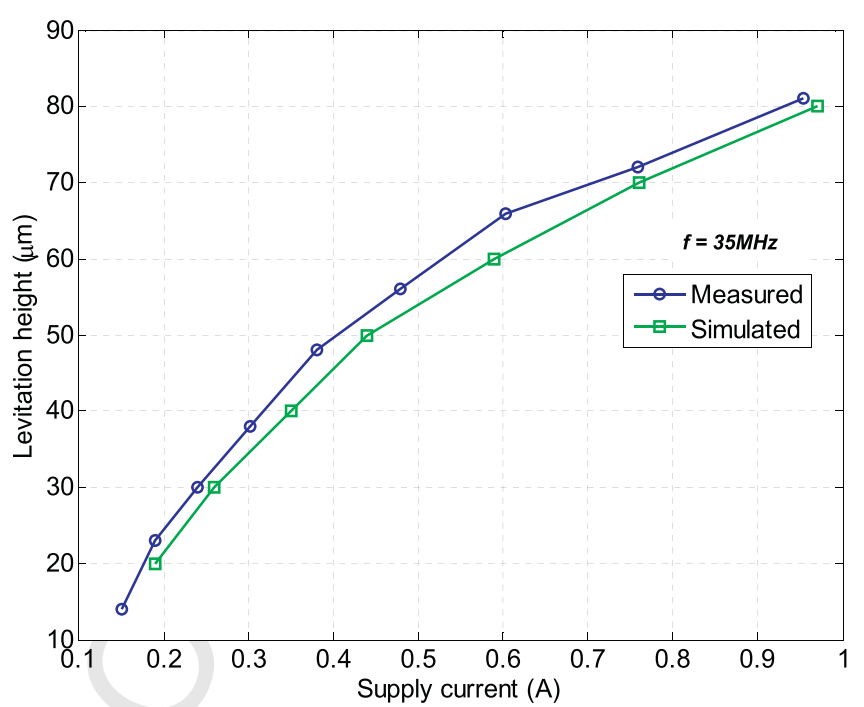

Fig. 13. Variation of levitation height with respect to the supply current at a frequency of $35 \mathrm{MHz}$. The levitation height increases with levitation current, but it has a decreasing slope.

had a phase difference of $180^{\circ}$. with respect to the neighboring electrodes pair. This maintained a continuous forward propulsion force to propel the object that depends on the frequency of the applied square wave. Using this circuit, the amplitude and the frequency of the square wave could also be independently controlled.

The average linear forward velocity was measured for different square wave propulsion voltage frequencies and is plotted in Fig, 15. These measurements were taken for a peak square wave voltage amplitude of $9 \mathrm{~V}$. The velocity increases linearly up to $1.5 \mathrm{~Hz}$, after which it starts decreasing with frequency. The forward motion of the micro-object is synchronized with the switching voltage up to its critical operation frequency. Above this frequency it cannot follow the voltage change due to its mechanical inertia and hence the forward velocity of the object decreases as the switching frequency is further increased. The maximum average linear forward velocity was measured to be $3.6 \mathrm{~mm} / \mathrm{s}$.

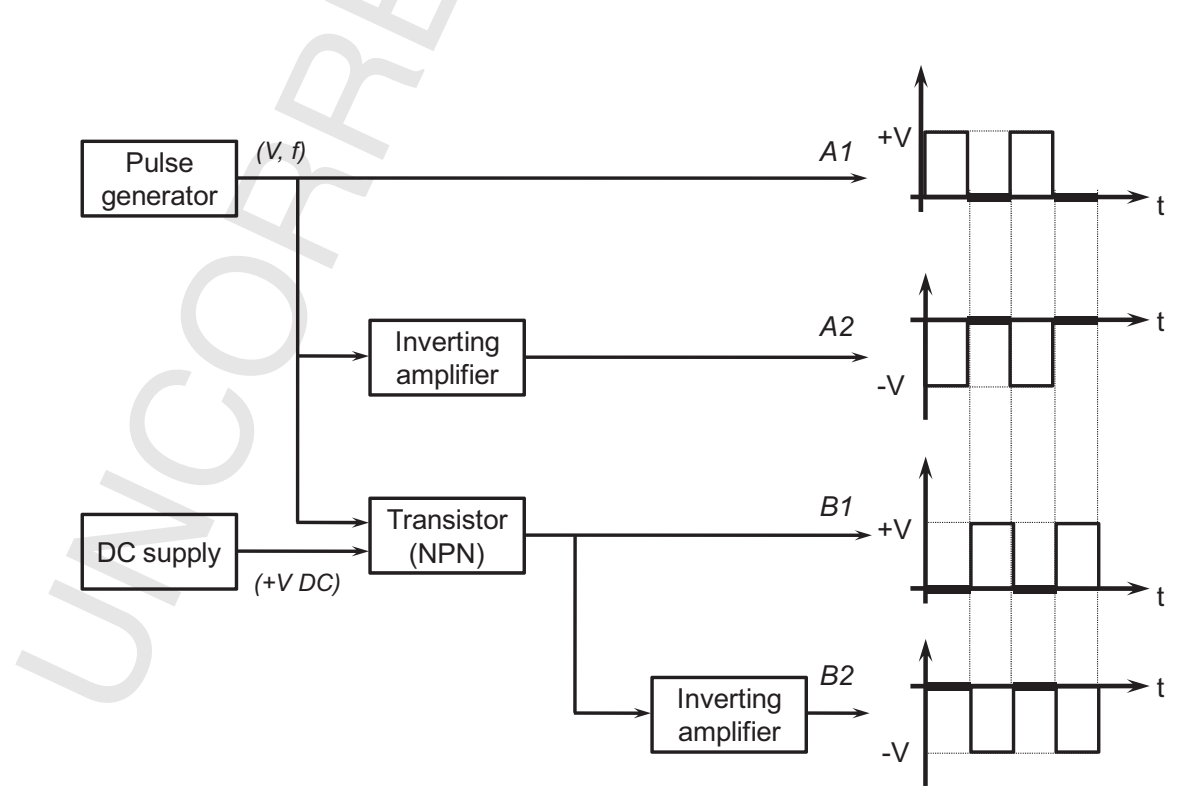

Fig. 14. Block diagram of the analog circuitry that is used to generate the sequential square waves for each pair of propulsion electrodes. 


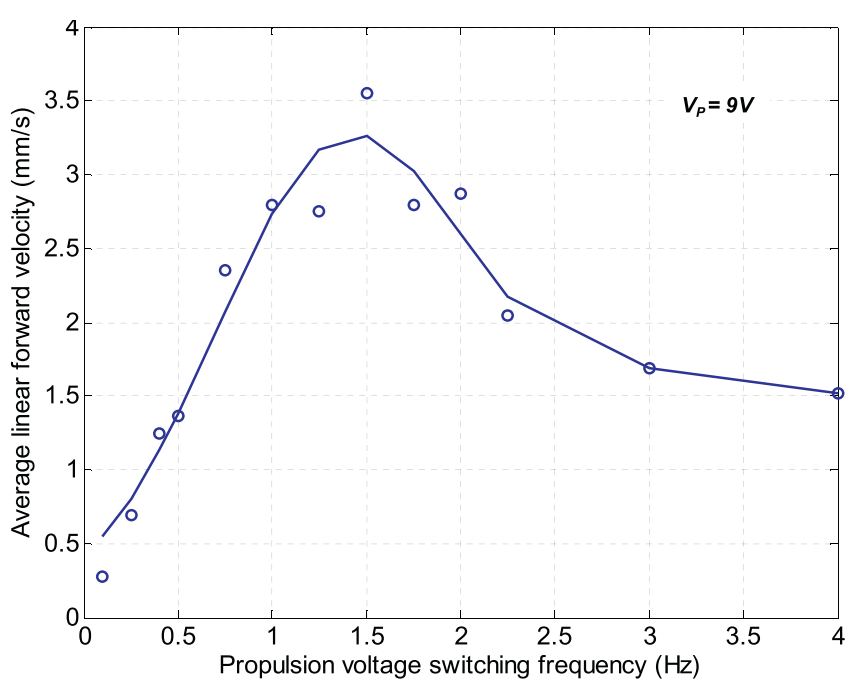

Fig 15. Average linear forward velocity with respect to the frequency of the propulsion voltage for a square wave voltage amplitude of $9 \mathrm{~V}$.

\section{Conclusion}

In this work, a micro-linear manipulator that can levitate and propel micro-objects is presented. The force on the micro-object was calculated by electromagnetic finite element FEM simulations in Comsol. The simulation model was based on the levitation and propulsion electrodes and the micro-object. However, in the actual experiment, the cabling, connections, and the substrate where the electrodes are fabricated, and the diameter of the wirebonds used are all important factors that can affect the maximum levitation force and frequency. For example, the Al wirebonds used in this work were $25 \mu \mathrm{m}$ in diameter and the frequency corresponding to $12.5 \mu \mathrm{m}$ skin depth that is half the wirebond diameter is $45 \mathrm{MHz}$ (see Fig. 5). This corresponds to the experimentally verified maximum levitation at $42 \mathrm{MHz}$. It is experimentally shown that by using the proposed technique, a micro-object could be levitated to a maximum levitation height of $75 \mu \mathrm{m}$ at $42 \mathrm{MHz}$ levitation frequency and propelled forward along a predefined linear path, at a maximum average linear forward velocity of $3.6 \mathrm{~mm} / \mathrm{s}$. The levitation height measurements were in good agreement with the simulations within an error margin of 5\%. It is observed that the forward propulsion velocity increases linearly with the frequency of the propulsion voltage and starts decreasing after $1.5 \mathrm{~Hz}$. This is because the object cannot follow the propulsion voltage after this frequency. For this reason, as a future work a continuous propulsion and an open ended design can be considered to increase the propulsion velocity and allow the micro-object to be shot off the device effectively realizing a micro-rail gun. Another improvement as a future work can be the investigation of the effect of damping forces over the performance of the proposed design. Damping forces like slide-film and squeeze film damping can become important depending on the objects velocity and separation between the object and the base. These effects have been omitted mainly because the FEM model was constructed and ran in the static mode in order to keep the model concise and also the main purpose of the study was to demonstrate that the object can be levitated and propelled forward using the proposed design. For this reason a detailed damping model will be investigated in an extended future study of the work. Such a damping model may be important in determining the maximum velocity of the object reached, which is $3.6 \mathrm{~mm} / \mathrm{s}$ in this specific work. The maximum velocity of the object may be too small to shoot it off to a meaningful distance if there exists an air damping force and/or gravity in the downward direction. However, the aim of this work was to show that using the proposed idea, an object can be levitated and moved forward in a contactless manner. In a future extended version, it is aimed to operate the proposed design in vacuum where there will be less counter air drag effects and hence a higher velocity can be reached.

The design has applications to levitate, position, and accelerate micro-objects for example to be used in contactless injection systems. It also has a future potential to form the basis of contactless chip positioning systems.

\section{References}

[1] I. Sari, M. Kraft, A micro electrostatic linear accelerator based on electromagnetic levitation, in: Proc. Transducers, June 2011, 2011, pp. 1729-1732.

[2] C. Shearwood, K.Y. Ho, C.B. Williams, H. Gong, Development of a levitated micromotor for application as a gyroscope, Sens, Actuators 83 (2000) 85-92.

[3] X. Wu, W. Chen, X. Zhao, W. Zhang, Development of a micromachined rotating gyroscope with electromagnetically levitated rotor, J. Micromech. Microeng. 16 (2006) 1993-1999.

[4] T. Murakoshi, Y. Endo, K. Fukatsu, S. Nakamura, M. Esashi, Electrostatically levitated ring-shaped rotational-gyro/accelerometer, Jpn J. Appl. Phys. 42 (2003) 2468-2472.

[5] W.S.H. Wong, W.K.S. Paot, D. Holliday, P.H. Mellor, Design and modeling of an electromagnetic levitated and actuated micromotor, in: IEEE International Conference on Semiconductor Electronics, Malaysia, 2006, pp. 309-313.

[6] B. Wagner, M. Kreutzer, W. Benecke, Permanent magnet micromotors on silicon substrates, J. Micromech. Microeng. 2 (March (1)) (1993) 23-29.

[7] G. He, K. Chen, S. Tan, W. Wang, Electrical levitation for micromotors, microgyroscopes and microaccelerometers, Sens Actuators A 54 (1996) 741-745.

[8] J.U. Jeon, T. Higuchi, Electrostatic suspension of dielectrics, IEEE Tran Ind. Electron. 45 (6) (1998) 938-946.

[9] V. Badilita, S. Rzesnik, K. Kratt, U. Wallrabe, Characterization of the 2nd generation magnetic microbearing with integrated stabilization for frictionless devices, in: Proc. Transducers, June 2011, 2011, pp. 1456-1459.

[10] S. Kumar, D. Cho, Electrostatically levitated microactuators, J. Micromech. Microeng. 2 (1992) 96-103.

[11] J. Jin, C.Y. Tachung, T. Higuchi, J.U. Jeon, Direct electrostatic levitation and propulsion of silicon wafer, IEEE Trans Ind. Appl. 34 (September-October (5)) (1998) 975-984

[12] W. Liu, W.Y. Chen, W.P. Zhang, X.G. Huang, Z.R. Zhang, Variable-capacitance micromotor with levitated diamagnetic rotor, Electron, Lett. 44 (11) (2008) 681-683.

[13] R.J. Hopkins, L. Mitchem, A.D. Ward, J.P. Reid, Control and characterisation of a single aerosol droplet in a single-beam gradient-force optical trap, Phys. Chem 6 (2004) 4924-4927.

[14] Microchemicals GmbH, http://www.microchemicals.eu/photoresist/ photoresist_az_nlof_2070_eng.htm

[15] Microchemicals GmbH, http://www.microchemicals.eu/remover_stripper html,

[16] Microchemicals GmbH, http://www.microchemicals.eu/photoresist/ photoresist_az_520d.html $\wedge$

\section{Biographies}

Ibrahim Sari is working at Southampton Nanofabrication Centre (SNC), Southampton, UK as the Commercial Processing Manager since 2014. He is managing and organizing the Nano- and Micro-fabrication focused commercial business activities of SNC. He has completed his Ph.D. at Middle East Technical University, Ankara Turkey in 2008 in the field of Micro-Electromechanical Systems (MEMS) based vibration energy harvesting. He has then joined University of Southampton, Electronics and Computer Science (ECS), Southampton, UK, as a Research Fellow and investigated novel techniques on levitation and propulsion of micro-objects using electromagnetic fields. In parallel to this role, he has developed novel MEMS fabrication techniques focusing on dicing free release and fabrication methods that can be used for the production of SOI-MEMS (Silicon-on-Insulator MEMS) inertial sensors. The techniques he has developed has been used in several academic and commercia projects to fabricate high-performance MEMS actuators and sensors. After completing his postdoctoral studies in 2012, he has started managing and organizing commercial projects carried out at the Cleanroom facilities of ECS department.

Michael Kraft works at the Fraunhofer Institute for Microelectronic Circuits and Systems in Duisburg, Germany. He heads the Department of Micro- and Nano-systems focussing on fully integrated microsensors and biohybrid systems. He concurrently holds the Professorial Chair of Integrated Micro- and Nano-systems at the University of Duisburg-Essen. From 1999 to September 2012, he has been a faculty member and Professor in Microsystemtechnology at the University of Southampton, UK. Concurrently, he also was the director of the Southampton Nanofabrication Centre. He graduated with a Dipl.-Ing. (Univ.) in electrical and electronics engineering at the Friedrich Alexander Universität Erlangen-Nürnberg in 1993. In 1997 he was awarded a PhD from Coventry University on the development of a MEMS accelerometer. He then spent two years at the Berkeley Sensors and Actuator Center at the University of California working on integrated MEMS gyroscopes. He has 20 years 
of experience in micro- and nano-fabrication techniques, microsensors and actuators and their interface circuits, in particular for capacitive sensors. He has a broad interest in MEMS and nanotechnology ranging from process development to system integration of MEMS and nano-devices. He has published over 200 peer reviewed journal and conference publications as an author or co-author. He also contributed to three text books on MEMS, and edited a book on MEMS for aerospace and automotive applications. He currently serves on several steering and technical committees of international conferences such as IEEE Sensors, Eurosensors, BioDevices and MME.
494

Please cite this article in press as: I. Sari, M. Kraft, A MEMS linear accelerator for levitated micro-objects, Sens. Actuators A: Phys. (2014), http://dx.doi.org/10.1016/j.sna.2014.11.008 